\title{
Solar powered oxygen systems in remote health centers in Papua New Guinea: a large scale implementation effectiveness trial
}

\author{
Trevor Duke $e^{1,2}$, Ilomo Hwaihwanje ${ }^{3}$, \\ Magdalynn Kaupa ${ }^{4}$, Jonah Karubi ${ }^{4}$, \\ Doreen Panauwe ${ }^{5}$, Martin Sa'avu' ${ }^{6}$, \\ Francis Pulsan 7 , Peter Prasad ${ }^{8}$, \\ Freddy Maru ${ }^{9}$, Henry Tenambo ${ }^{10}$, \\ Ambrose Kwaramb ${ }^{10}$, Eleanor \\ Neal $^{1}$, Hamish Graham ${ }^{1}$, Rasa \\ Izadnegahdar $^{11}$
${ }^{1}$ Center for International Child Health, University of Melbourne and MCRI, Melbourne, Australia
2 School of Medicine and Health Sciences, University of PNG, Taurama Campus, NCD, Papua New Guinea
${ }^{3}$ Goroka General Hospital, Eastern Highlands Province, Goroka, Papua New Guinea
${ }^{4}$ Mt Hagen General Hospital, Western Highlands, Mount Hagen, Papua New Guinea
${ }^{5}$ Wabag Hospital, Enga Province, Wabag, Papua New Guinea
${ }^{6}$ Mendi Hospital, Southern Highlands Province, Mendi, Papua New Guinea
${ }^{7}$ School of Medicine and Health Sciences, University of PNG, Taurama Campus, NCD, Papua New Guinea
${ }^{8}$ AusTrade Pacific, Sydney, Australia
${ }^{9}$ AusTrade Pacific, Port Moresby, Papua New Guinea
${ }^{10}$ Health Facilities Branch, National Department of Health, Papua New Guinea
${ }^{11}$ Bill and Melinda Gates Foundation, Seattle, Washington, USA

\section{Correspondence to:}

Trevor Duke, MD FRACP FCICM

Royal Children's Hospital

50 Flemington Road

Parkville

Victoria, 3052

Australia

trevor.duke@rch.org.au
Background Pneumonia is the largest cause of child deaths in Papua New Guinea (PNG), and hypoxaemia is the major complication causing death in childhood pneumonia, and hypoxaemia is a major factor in deaths from many other common conditions, including bronchiolitis, asthma, sepsis, malaria, trauma, perinatal problems, and obstetric emergencies. A reliable source of oxygen therapy can reduce mortality from pneumonia by up to $35 \%$. However, in low and middle income countries throughout the world, improved oxygen systems have not been implemented at large scale in remote, difficult to access health care settings, and oxygen is often unavailable at smaller rural hospitals or district health centers which serve as the first point of referral for childhood illnesses. These hospitals are hampered by lack of reliable power, staff training and other basic services.

Methods We report the methodology of a large implementation effectiveness trial involving sustainable and renewable oxygen and power systems in 36 health facilities in remote rural areas of PNG. The methodology is a before-and after evaluation involving continuous quality improvement, and a health systems approach. We describe this model of implementation as the considerations and steps involved have wider implications in health systems in other countries.

Results The implementation steps include: defining the criteria for where such an intervention is appropriate, assessment of power supplies and power requirements, the optimal design of a solar power system, specifications for oxygen concentrators and other oxygen equipment that will function in remote environments, installation logistics in remote settings, the role of oxygen analyzers in monitoring oxygen concentrator performance, the engineering capacity required to sustain a program at scale, clinical guidelines and training on oxygen equipment and the treatment of children with severe respiratory infection and other critical illnesses, program costs, and measurement of processes and outcomes to support continuous quality improvement.

Conclusions This study will evaluate the feasibility and sustainability issues in improving oxygen systems and providing reliable power on a large scale in remote rural settings in PNG, and the impact of this on child mortality from pneumonia over 3 years post-intervention. Taking a continuous quality improvement approach can be transformational for remote health services.

Pneumonia is the largest cause of child deaths in Papua New Guinea (PNG), as it is globally $[1,2]$. Hypoxaemia is the major complication causing death in childhood pneumonia and is highly prevalent in highlands region of 
PNG. In provincial hospitals in PNG the mortality rate for pneumonia and severe pneumonia are 5\% and $10 \%$, respectively [2]. In previous studies in PNG we showed that improved oxygen systems, which include a reliable source of oxygen therapy using concentrators, and pulse oximetry for detection of hypoxaemia, can reduce mortality from pneumonia by up to 35\% [3]. Hypoxaemia occurs in many other common conditions: bronchiolitis, sepsis, asthma, tuberculosis, HIV-related lung disease, malaria, perinatal problems, trauma, obstetric emergencies, and chronic respiratory and cardiac disease in adults and children [4-7]. A reliable oxygen system is therefore a critical minimum input for adequate health care in all settings where such emergencies present and such conditions are managed.

However, in PNG and in low and middle income countries throughout the world, improved oxygen systems have only been implemented where it is relatively easy to do so; in larger provincial or referral hospitals with adequate power supplies and staff capacity $[3,8,9]$. In these countries, including those with the largest shares of the global burden of childhood pneumonia deaths such as Ethiopia and PNG, a high proportion of the population live in rural areas, where access to urban centers and referral health care facilities is limited or non-existent. Improved oxygen systems have not been implemented at scale in such settings, and do not reach smaller rural hospitals or district health centers that serve as the first point of referral for acute illness. Many children with severe pneumonia present to these small rural hospitals and district health centers where hypoxaemia often goes undetected or untreated. Barriers to improving oxygen systems in these settings include the lack of adequate power, uncertain long-term reliability of equipment in remote settings, logistics of implementation, lack of preventive maintenance, lack of recognition of the importance of oxygen or identification of hypoxemia using pulse oximetry, limited training of health workers in the recognition of hypoxaemia, and lack of financial investment in quality health services in rural and remote areas. Oxygen concentrators have previously been run using solar power, but only on a small scale in a single health facility [10,11].

We report the steps needed to implement basic oxygen systems in 36 health facilities in remote rural areas of PNG. The decisions and steps involved included: defining the criteria for facilities where such an intervention is appropriate, assessment of power supplies and power requirements, the optimal design of a solar power system that can address both oxygen equipment load and for other essential health facility equipment, specifications for oxygen concentrators and other oxygen equipment that will function in remote and tropical environments, implementation logistics in remote settings, the role of oxygen analyzers in monitoring oxygen concentrator performance, the engineering capacity required to sustain a program at scale, clinical guidelines and training on oxygen equipment and the treatment of children with severe respiratory infection and other critical illnesses, program costs, and measurement of processes and outcomes to inform continuous quality improvement.

These steps to scaling up oxygen systems in the most challenging environments have not previously been actioned in a large field trial. We describe this model of implementation in the hope that other countries will build on this.

\section{The project}

Previous research has been conducted to understand the epidemiology of hypoxaemia in PNG $[4,12,13]$, the quality of care provided in rural and district hospitals [14], and the effect of oxygen on case fatality rates from pneumonia [3]. What has not been demonstrated until now is the sustainable implementation at large scale in the most difficult environments, and the steps required to achieve this. This project is funded by the Bill and Melinda Gates Foundation and other partners. Project funding, which also included support for trialing the implementation of oxygen concentrators in 12 urban hospitals in Nigeria [15], was provided in 2014. Preparatory work for this project occurred in 2014 and 2015.

\section{METHODS}

\section{The process for identifying suitable facilities}

Criteria for selecting suitable health facilities was determined by wide stakeholder consultation between representatives of the National Department of Health, provincial health, nurses and doctors, administrators and technicians. Broad principles for selection were set out and suitability was ascertained by on-site assessments and review of the available routine data. Selection of participating health facilities was on the basis of clinical need and the capacity for benefiting that community. The need for sources of oxygen and 
power are high for health facilities that are located within large population areas (even if that population is dispersed over a wide area) where the prevalence of pneumonia and other acute respiratory infections is high, and where access to a provincial referral hospital is limited or transportation is likely to be hazardous for seriously ill patients. Other principles for health facility selection were that the health facility had difficulty gaining access to a reliable source of oxygen; that the facility was open and functioning; and staff were motivated to participate. In PNG while some primary health centers are closed or not functioning, many, particularly district health centers fulfilled these criteria, are functioning to the best of their capacity and have staff who are enthusiastic and committed.

Baseline data were collected on all proposed health facilities. This involved routine recording of admissions, deaths and referrals for the previous 3 years (Table 1 ).

Data were also collected on characteristics of the health services: the number of health personnel, levels of training and recent continuing education, health facility bed capacity for children, availability of oxygen equipment, existing power sources, security issues at the health facility, and essential medicines, equipment, commodities and procedures for infection control, and laboratory tests.

\section{Assessment of power supplies and power requirements}

We have previously assessed the power supplies in five district hospitals using an Electrocorder [14]. This demonstrated that even in facilities that are connected to mains power, power supplies are erratic, with many outages, fluctuations and surges. These abrupt changes in power have the high likelihood of damaging oxygen concentrators and other equipment, and may be one of the key reasons for concentrator failure in previous studies. In this current field trial we did not reproduce Electrocorder readings as the health facilities included were at an even lower level than the previously assessed 5 district hospitals, and were either without mains power, with known unreliable power, or with an alternative source of renewable power, such as hydropower. If a health facility only had a diesel backup generator this was not considered sufficient, as the costs, availability and logistics of diesel fuel are an obstacle to using electrically powered equipment.

\section{The optimal design of a solar power system}

Considerations in the design of the solar panel included the efficiency of the system, the peak sun hours for the specified tilt angle (4.53 hours), a 25\% oversupply of power, and the total daily energy demand. The system was designed to supply $11.44 \mathrm{kWh} / \mathrm{d}$ daily average load, using eighteen $240 \mathrm{~W}$ panels. This was based on the power requirements for a rural health facility for treating common childhood illnesses [16]. A battery backup system was designed to provide 3 days autonomy at $80 \%$ depth of discharge. Other solar equipment calculations are included in Online Supplementary Document.

\section{Specifications for oxygen concentrators and other oxygen equipment that will function well in remote environments}

The choice of oxygen equipment was based on over a decade of trial and error, in PNG and in other countries $[8,17-20]$. The principles are that concentrators needed to be robust, standardized, and able to function well at high temperature and high humidity, and have a relatively low power requirement. Concentrator specifications follow the now published WHO guidelines for oxygen concentrators [21]. We use Airsep Elite 5 1/min concentrators (Chart Industries, New York NY, USA) and Lifebox pulse oximeters (Lifebox, London, UK), both of which have been successfully used in larger hospitals in previous field trials in PNG and other developing countries. Busy district hospitals are receiving three concentrators, and smaller health centers are receiving two. Theoretically, several patients can be treated at any one time

Table 1. Baseline data in 36 health centers and district hospitals

\begin{tabular}{|c|c|c|c|c|c|c|c|c|c|c|}
\hline Year & $\begin{array}{l}\text { PaEdiatalC } \\
\text { aDMISSIONS }\end{array}$ & $\begin{array}{c}\text { Paediatric } \\
\text { DeATHS }\end{array}$ & CFR (\%) & Peneumonia & $\begin{array}{c}\text { Puevmonia } \\
\text { deathS }\end{array}$ & $\begin{array}{l}\text { Pueumonia } \\
\text { CFR (\%) }\end{array}$ & Births & NND & $\begin{array}{c}\text { NMR } \\
\text { (PER 1000) }\end{array}$ & $\begin{array}{l}\text { ChILDREN } \\
\text { referred out }\end{array}$ \\
\hline 2014 & 8511 & 309 & 3.63 & 3510 & 146 & 4.16 & 10933 & 98 & 9.0 & 687 \\
\hline 2013 & 7193 & 236 & 3.28 & 2849 & 125 & 4.39 & 10329 & 88 & 8.5 & 695 \\
\hline 2012 & 5104 & 216 & 4.23 & 2447 & 86 & 3.51 & 10284 & 84 & 8.2 & 791 \\
\hline Total & 20808 & 761 & 3.66 & 8806 & 357 & 4.05 & 31546 & 270 & 8.6 & 2173 \\
\hline
\end{tabular}

NND - neonatal deaths, CFR - case fatality rate 
using a concentrator using a flow meter assembly which splits flow to several patients (Sureflow meter, Airsep FM069-1, Chart Industries, Galveston USA). Typically two patients at any one time can be safely managed using one concentrator, even if they are each severely hypoxic and requiring standard flow rates of $2 \mathrm{~L}$ min. Sometimes very unwell children will require higher flows in the initial treatment period and may need a single concentrator $[19,22]$.

\section{Installation logistics}

This project involves implementation teams and provincial monitoring teams. Implementation consisted of several skilled teams of $4-5$ workers. The team leaders were trained in installing the solar power system, and in commissioning and use of oxygen concentrators. Large transport logistics were required to haul equipment from wharf up the PNG highlands highway, and smaller vehicles were required on unmade feeder roads to remote health centers. The installations were preceded by site visits, to discuss with the community and health workers, to ensure facilities fulfilled the criteria for inclusion, and to plan the siting of solar panels for maximal effectiveness. The installations typically took 2-3 days in each center, occurring between March and October 2016.

The implementation teams followed up to assess whether the equipment was being properly used and cared for.

\section{Monitoring oxygen concentrator performance using oxygen analysers}

Health care workers, particularly in remote settings, have an important role in maintaining equipment. For decades many oxygen concentrator projects have failed the test of even medium term sustainability because of technical faults that were detected late or not at all, leading to entire oxygen system failure. In most health facilities throughout the world qualified engineers do not monitor equipment regularly (as engineers may be stationed at a major hospital rather than a district hospital or health center). Therefore preventative maintenance of oxygen concentrators (apart from occasional external filter cleaning) is limited or non-existent. Use of simple oxygen analyzers (Maxtec $\mathrm{O}_{2}$ analyzer, Maxtec, Utah USA) by health care workers to monitor the performance of concentrators (fraction of oxygen produced, to ensure it is $>85 \%$ ), and the flow rate, and training health workers to understand the meaning of alarms on the concentrator will enable early detection of developing faults or parts wearing, and early communication with provincial engineers. Given the limits of current concentrator technology this is a key to addressing the problems of all previous research in concentrators in low resource settings. We taught all health staff how to use oxygen analyzers and provided one for each health facility.

\section{The engineering capacity required to sustain a program at scale}

Previous oxygen projects have had centralized engineering capacity, but these have floundered because technicians change roles, move into the private sector, or because of over-reliance on one or two busy persons. In this project we have decentralized the technical capacity as much as possible, by optimizing what nurses in health centers can do in preventative maintenance and monitoring of equipment performance, and training provincial and district level technicians, and having spare parts available in provincial locations which are at most 2-5 hours' drive away.

\section{Clinical guidelines and training on oxygen equipment and the treatment of children with severe respiratory infection and other critical illnesses}

We use the WHO guidelines for the Clinical Use of Oxygen in Children [22], and the WHO Hospital Care for Children for training [23]. The training is practical, initially workshop based, and repeated on several occasions and reinforced on health center visits by the provincial pediatricians. The training combines clinical and technical teaching and experience, and the messages are heard in more than one forum and more than one way - direct facilitator led teaching, peer and facilitator supervised practical examples, breaking complex tasks and skills into component parts and modeling each component, and presenting a holistic approach to the management of sick children. Training covers an understanding of hypoxaemia in pneumonia, other acute respiratory infections and non-respiratory diseases, respiratory clinical signs, principles and practical use of pulse oximetry, oxygen concentrators and how they work, preventative maintenance and trouble-shooting, use of oxygen analysers for assessing performance of concentrators, safe use of oxygen therapy in newborns, resuscitation, and the concept and practical aspects of continuous quality improvement. The curriculum is based on the WHO guidelines [22]. 
For many health care workers, the health technologies we introduce are new and sometimes daunting. Oxygen concentrators, like all technologies, may fail because people cannot understand them, or are wary of them, or cannot competently perform the practical tasks of checking and maintenance. A considerable part of this project on the sustainability of better oxygen systems in remote low resource settings involves a novel technology-clinical interface. Until now, these health care settings have been a technology-free zone and the integration of technology into clinical medicine in these settings is unproven and experimental. The elements of a successful technology-clinical interface, if it can be achieved, need to be better understood. If health care workers and technicians have not physically performed a given procedure during training, it is not likely they can do it in their own health care setting (eg, changing oximeter probes, replacing oximeter batteries, replacing concentrator filters, testing concentrator alarms and using an oxygen analyzer). Clinical and technical training tools are available at http://www.hospitalcareforchildren.org/.

\section{Program costs}

It is relatively inexpensive to provide oxygen concentrators, however solar power is expensive. The cost per health facility is approximately US\$50000, including oxygen and solar equipment, installation and logistics, and training. Over the course of the formal training, on-site support and continuous quality improvement, all health workers who manage children in these 36 facilities will have training and hands on assessment of skills.

\section{RESULTS}

\section{Baseline data on logistics and health services}

Of 36 health facilities originally proposed, 6 were unsuitable because of poor road conditions that would not allow a vehicle carrying equipment to access the health center $(n=3)$ or the health center was in a state of disrepair $(n=3)$. Six more health facilities which fulfilled the criteria were selected to replace these. The catchment population of these 36 health facilities was 1223755 in the last (2011) National census, out of a total PNG population of 7.013 million. The median number of beds for children was 10 (interquartile range IQR 5-13), and newborns was 4 (0-8). Only 10 of the 36 health facilities have doctors. The median number of nurses on staff was 4 (IQR 2-10), and 29 had one or more midwives (12 health facilities had only one midwife). Only 16 of the health facilities had a trained pediatric nurse, with the other 20 having general trained nurses. Community health workers were present in all health facilities, with the median number of 7 (IQR 3-17). Only 16 health facilities were connected to mains power, 21 had a petrol generator. Running water was available in 30 health facilities (83\%). Most health facilities had simple antibiotics for the treatment of pneumonia, either amoxycillin $(n=33)$ or penicillin (all health facilities). 34 had vaccine refrigerator, and most were stocked with vaccines: measles vaccine $(n=34)$, Pentavalent (32), but BCG was less available ( 25 health facilities). All but one had infant weighing scales and safe sharps disposal, but a minority (10) could check blood glucose, hemoglobin (10), or had x-ray facilities (7). Malaria tests were available in 28 and sputum smear for tuberculosis in 20.

\section{Baseline data on outcomes}

In the 36 participating health centers we gathered data on over 20000 admissions in the 3 years prior to the installations (Table 1). Over 8000 admissions were for pneumonia. The overall case fatality rate was $3.7 \%$ and the case fatality rate for pneumonia was $4.1 \%$. Just over $10 \%$ of admissions required referral to provincial hospitals. These baseline data were collected from health facility admission record books, which are historically meticulously kept by nursing staff in PNG.

A sample size calculation was based on the primary outcome of reducing pneumonia mortality - from $4 \%$ to $3 \%$, with $90 \%$ power $=7295$ in each arm. The study was also adequately powered to detect a different of $20 \%$ in overall mortality rate, and a $20 \%$ reduction in referrals to tertiary centers. Other outcomes are listed in Table 2.

\section{Assessing continuous quality improvement outcomes}

Continuous quality improvement (CQI) involves regular monitoring and supervision by provincial supervisory teams; each team consists of the provincial pediatrician and a technician trained in oxygen and solar equipment. These reviews will occur every 4 months after implementation, will be carried out by 
Table 2. Outcomes, research questions, sources of data and specific metrics

Dutenme catiegory Soung

1 Deaths from pneumonia; Is there a difference in pneumonia case Admission record books Pneumonia case fatality rate: Pneumonia overall pediatric deaths fatality rates and overall pediatric CFR in health facility from pre to post implementation of improved oxygen therapy and solar power?

$\begin{array}{ll}2 \text { Referral / transfer } & \begin{array}{l}\text { Is there any difference in referral rates Admission record books } \\ \text { from pre to post intervention? }\end{array}\end{array}$

2 Referral / transfer $\quad \begin{aligned} & \text { Is there any difference in referral rates Admission record books } \\ & \text { from pre to post intervention? }\end{aligned}$ deaths / pneumonia admissions (\%). Paediatric case fatality rate: Paediatric deaths / all pediatric admissions (\%).

3 Patient characteristics What are the conditions associated with Standardised admission re- Diagnoses associated with hypoxaemia (proand response to oxygen hypoxaemia in remote rural health facili- cord data portions) disaggregated for neonates and chiltherapy - effectiveness of ties? What is the response to oxygen theroxygen therapy using the apy when oxygen is given using solarmethod we have de- powered oxygen concentrators? What is signed to treat hypoxae- the duration of hypoxaemia in children mia managed in remote rural health facilities? dren $>1$ mo. Response to oxygen therapy (median change in $\mathrm{SpO}_{2}$ in the first 30 min; and proportion responded / not responded, ie, proportion with persisting $\mathrm{SpO}_{2}<90 \%$, or severe signs of respiratory distress $30 \mathrm{~min}$ after commencing oxygen). Days of oxygen therapy. Duration of hypoxaemia for neonates and children $>1$ month.

4 Maintenance of oxygen Are concentrators maintained well, are Oxygen concentrator per- What proportion of oxygen concentrators unequipment problems identified and is appropriate ac- formance log-books. On- dergo weekly maintenance and performance tion taken? What proportion of concentra- site checks on support and checking? List of problems identified and actors undergo weekly maintenance and monitoring visits tion taken. Number/proportion of concentraperformance checking? What problems are identified? What proportion of concentrators are functioning well after 1,2 tors providing $>85 \%$ oxygen and reliable flow rates as checked by oxygen analyzer at 1,2 and and 3 years since installation?

5 Health workers knowl- What is the oxygen knowledge and skill Oxygen competency tests - Repeating oxygen knowledge and skill tests at edge and skill of oxygen of the health workers in remote health repeated measures $\quad 12$ monthly intervals. therapy facilities? Does this improve with training and CQI

6 Reliability, efficiency and Solar power output (quantitative kW Tristar 60 Amp controller adequacy of solar power hours per day), adequacy of this power for running concentrators and other equipment needed by the health facility, and any problems identified.

\begin{tabular}{lll}
\hline 7 Training outcomes & $\begin{array}{l}\text { What training was done and what are the } \\
\text { perceived training needs? }\end{array}$ \\
\hline 8 Sustainable processes & $\begin{array}{l}\text { Is it sustainable in PNG to have concen- } \\
\text { trators run off solar power as the source sour } \\
\text { of oxygen in remote areas? }\end{array}$
\end{tabular}
Project records Mixture of the above data $\begin{array}{ll}\begin{array}{l}\text { trators run off solar power as the source } \\ \text { of oxygen in remote areas? }\end{array} & \text { tion of relevant events, } \\ \text { meetings and occurrences }\end{array}$ $\begin{aligned} & \text { trators run off solar power as the source sources, and documenta- } \\ & \text { of oxygen in remote areas? } \\ & \text { tion of relevant events, } \\ & \text { meetings and occurrences }\end{aligned}$ $\begin{aligned} & \text { trators run off solar power as the source sources, and documenta- } \\ & \text { of oxygen in remote areas? } \\ & \text { tion of relevant events, } \\ & \text { meetings and occurrences }\end{aligned}$

Solar power output: average $\mathrm{kW}$ hours per day produced by each solar system in the previous 6 mo. Occurrence of breakdown of solar output, such that concentrators are unable to function.

Record of training courses conducted: formal; in-house as part of CQI How would we measure sustainability? Equipment sustainability (ie, proportion of concentrators and oximeters still functioning well at 1, 2, 3 years). Energy sustainable (proportion of solar power systems still functioning well at 1, 2, 3 years). Health worker sustainability (proportion with good oxygen knowledge and skills at 1, 2, 3 years). Financial sustainability (examples where national, provincial or local governments have invested in other facilities in a similar way). Policy sustainability (occurrence of a national oxygen policy that incorporates these principles). Documentation of other examples of engagement by policy makers.

9 Wider benefits Does CQI in rural health facilities im- Above data and qualitative prove wider outcomes? Care seeking by assessments parents? Health worker morale?

are seeking: Number of children brought to health facilities (admission activity number per year for 3 years, compared with pre-intervention baseline data). Women having confidence in the health services: Number of babies born at health facilities (births per year for 3 years, compared with pre-intervention baseline data). Qualitative assessment of what health workers think of the oxygen project, whether and how it helps them in their work, what problems it may have resulted in, what could be done to help them better serve their communities, how they feel about their work, what their hopes are for their careers.

$\mathrm{CQI}$ - continuous quality improvement, $\mathrm{CFR}$ - case fatality rate, $\mathrm{SpO}_{2}$ - arterial oxygen saturation 
the provincial teams and will include on-site training, collection of data on functioning of equipment, utilization, maintenance needs, and trouble-shooting of problems identified. There will be multiple assessments during these visits over the 3 years, including for example, whether nurses can accurately use oxygen analysers to effectively monitor the performance of concentrators, and whether the solar power capacity is sufficient for energy demand. The CQI approach involves feedback by the provincial monitoring teams - feedback is to the health center staff, the district and provincial authorities, and the National Department of Health. CQI outcomes also include whether interventions occurs in response to feedback, and the effect of these responsive interventions. An assessment of sustainability processes, including policy maker engagement, and the wider effects of this project on care seeking, health facility functioning and health worker morale is under way (Table 2).

\section{DISCUSSION}

Over the past two decades mortality from childhood pneumonia has decreased significantly. While much effort has been put into vaccines and first line antibiotics for pneumonia, there is still a need for scaling up broader prevention and treatment initiatives. Even if severe pneumococcal infection could be markedly reduced by vaccination, realistically there will still be a myriad of respiratory viruses, particularly respiratory syncytial virus, and other viruses such as influenza, human metapneumo virus, parainfluenza; non-vaccine type pneumococci and Haemophilus; tuberculosis and other bacteria, which cause lower respiratory tract infections and fill up hospitals in low- and middle-income countries, as is the case in developed countries. The residual mortality, regardless of etiology will likely rest in those with more than a need for antibiotics alone. The population will likely be a more complex, comorbid, undernourished population where supportive care is increasingly important, including a role for oxygen therapy. The challenge of how to provide services in primary care and regional centers is not unique to PNG or to even to developing countries, the same drive to improve quality and reduce the need for referral is a focus in wealthy countries also. But the challenges are greater in rural, low income settings.

This implementation paper documents the steps to scaling up oxygen therapy in remote rural settings. Many steps are involved and this is a complex health care intervention. Previously oxygen has been thought of as an essential drug [24], but it is also an essential service. With the drive to increase facility births in low income, high mortality countries, health facilities have to reach a certain standard to signal to the community that they can provide a greater level of service than delivering at home - these essential services include power, clean water, oxygen, and infection control and prevention. As more and more births in countries like India, Ethiopia and Nigeria are happening in health facilities, there has been an ecological and foundational shift in care at these facilities. It is important to understand the importance of oxygen as a relatively simple yet essential service to maintain a minimum standard of quality in the redefined roles of primary health care and first referral facilities. Other investments, such as building sleeping quarters for staff to allow them to stay overnight, have to be prioritized also, and where oxygen, power, and running water fit into these priorities is a matter of judgement, but they all can be regarded as essential. PNG still has low rates of facility delivery (40\%), but further increases will require explicit attention on ensuring that minimum services are in place close to where communities live.

We emphasize the importance of a holistic health systems approach, community engagement, and continuous quality improvement. To be sustainable and maximally effective such interventions should integrate into routine health system functioning, and the benefits must be cross-cutting for maximum adoption and effect. For example, there is limited value in installing solar power to run an oxygen concentrator if there is no light in the delivery room, as women will not want to deliver babies overnight. Lack of basic services effect the health center's reputation and have adverse effects on community demand and care seeking (such as when an infant is unwell with pneumonia). However addressing such conditions, for example by providing power to run a light, and the vaccine refrigerator, and oxygen, can be transformational $[16,25]$. To be maximally efficient, such interventions should be based on a holistic and systematic approach to improving health service quality, with the provision of basic oxygen technology and adequate power as an entry point. Along with transforming a community's perception of a health center, essential services like oxygen and a reliable power system, and training to match, can boost health workers confidence and morale.

PNG is an expensive place to do such work, as the terrain is difficult, and distances far. Many concentrator programs only report the oxygen concentrator costs, but we have previously shown that the implementation and training costs are at least as much again as the costs of concentrator and other oxygen 
equipment [1]. Sustainability and expansion of such programs require an accurate estimation of true costs and benefits, so that health administrators and governments can order priorities according to their needs and program according to their available resources. In a decentralized system where provinces and districts have to decide on their health spending priorities, this project leaves no doubt as to the full costs of such a health facility service upgrade. There are many health priorities for child health in the sustainable development era, including health facility quality improvement, and each have real costs that have to be weighed [26].

Over the next 3 years we will measure clinical effectiveness and what is required to sustain this program, from simple things like whether nurses can accurately use oxygen analysers to effectively monitor the performance of concentrators, to whether the solar power capacity is sufficient for energy demand. And we will monitor, beyond effectiveness in the field, the administrative and political steps required for decisions about involvement of other provinces in the program, and how such interventions can be seen as cross-cutting, and part of a greater whole - quality services at district hospitals and rural health centers. A serious problem for many such rural health services and primary health care workers in the past has been low expectations, and showing that appropriate and essential technology such as this can be effective and sustained may just raise expectations to the level needed in the era of sustainable development.

\section{CONCLUSIONS}

This study will evaluate the feasibility and sustainability issues in improving oxygen systems and providing reliable power on a large scale in remote rural settings in PNG, and the impact of this on child mortality from pneumonia over 3 years post-intervention. Taking a continuous quality improvement approach may be transformational for remote health services.

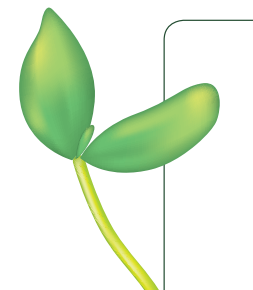

Acknowledgments: We thank the Bill and Melinda Gates Foundation for funding, which helped support the salaries of HG, EN and FM. The salaries of other authors were provided by the National Department of Health or PNG Provincial Health services, as part of their routine roles. We thank the members of the Family Health Services Branch of the PNG National Department of Health, and the nurses and doctors who work in each of the 36 health facilities involved in this project. We thank the RE Ross Trust for funding for training.

Ethics: The trial was approved by the PNG National Department of Health, The Human Research Ethics Committee of the University of Melbourne (1543797.1), the Provincial Health authorities in each participating province, and Church Health Services which run some of the participating health facilities. The project is registered by Australian New Zealand Clinical Trials Registry: ACTRN12616001469404. No individual patients were recruited to this study, the data collection is based on routine reporting, so no consent was required by the ethical review committees.

Funding: This project is funded by the Bill and Melinda Gates Foundation. Project funding, which also included support for trialing the implementation of oxygen concentrators in 12 urban hospitals in Nigeria, was provided in 2014. Preparatory work for this project occurred in 2014 and 2015. Additional funding was provided by the RE Ross Trust (Victoria) for training aspects, and by the National Health Department of PNG, and the Provincial Health Authorities in each province (in-kind funding with contributions by many clinical and technical staff). The funding agencies, especially the Bill \& Melinda Gates Foundation and the PNG National Department of Health, have worked closely with the project leaders, with input into design and method of analysis, but the final methodology, analysis and decision to publish are independent of the funding agencies.

Availability of data and materials: The baseline data (all that is summarized in this report) are fully available from the authors on request. Further details of technical information which are summarized in this report are available in Online Supplemetary Document.

Authors' contributions: All authors contributed substantially to several aspects of this study. TD designed the project in collaboration with IH, JK, MK, DP, MS and FP who led the clinical training and evaluation in respective provinces. FM, PP lead the equipment installation teams, supported by HT and AK. EN, HG RI supported the design and administrative aspects of the project. The paper was written by TD, with input from IH, MK and RI. All authors reviewed and approved the final manuscript.

Competing interests: The authors completed the Unified Competing Interest form at www.icmje.org/coi_disclosure.pdf (available upon request from the corresponding author), and declare no conflict of interest. 
1 Liu L, Oza S, Hogan D, Perin J, Rudan I, Lawn JE, et al. Global, regional, and national causes of child mortality in 2000_ 13, with projections to inform post-2015 priorities: an updated systematic analysis. Lancet. 2015;385:430-40. Medline:25280870 doi:10.1016/S0140-6736(14)61698-6

2 Duke T, Yano E, Hutchinson A, Hwaihwanje I, Aipit J, Tovilu M, et al. Large-scale data reporting of paediatric morbidity and mortality in developing countries: it can be done. Arch Dis Child. 2016;101:392-7. Medline:26489801 doi:10.1136/archdischild-2015-309353

3 Duke T, Wandi F, Jonathan M, Matai S, Kaupa M, Sa'avu M, et al. Improved oxygen systems for childhood pneumonia: a multihospital effectiveness study in Papua New Guinea. Lancet. 2008;372:1328-33. Medline:18708248 doi:10.1016/ S0140-6736(08)61164-2

4 Duke T, Blaschke AJ, Sialis S, Bonkowsky JL. Hypoxaemia in acute respiratory and non-respiratory illness in neonates and children in a developing country. Arch Dis Child. 2002;86:108-12. Medline:11827904 doi:10.1136/adc.86.2.108

5 Graham SM, Mtitimila EI, Kamanga HS, Walsh AL, Hart CA, Molyneux ME. Clinical presentation and outcome of Pneumocystis carinii pneumonia in Malawian children. Lancet. 2000;355:369-73. Medline:10665557 doi:10.1016/S01406736(98)11074-7

6 Subhi R, Adamson M, Campbell H, Weber M, Smith K, Duke T, et al. The prevalence of hypoxaemia among ill children in developing countries. Lancet Infect Dis. 2009;9:219-27. Medline:19324294 doi:10.1016/S1473-3099(09)70071-4

7 Mwaniki MK, Nokes DJ, Ignas J, Munyoki P, Ngama M, Newton CRJC, et al. Emergency triage assessment for hypoxaemia in neonates and young children in a Kenyan hospital: an observational study. Bull World Health Organ. 2009;87:26370. Medline:19551234 doi:10.2471/BLT.07.049148

8 Enarson P, La Vincente S, Gie R, Maganga E, Chokani C. Implementation of an oxygen concentrator system in district hospital paediatric wards throughout Malawi. Bull World Health Organ. 2008;86:344-8. Medline:18545736 doi:10.2471/ BLT.07.048017

9 Dobson M, Peel D, Khallaf N. Field trial of oxygen concentrators in upper Egypt. Lancet. 1996;347:1597-9. Medline:8667871 doi:10.1016/S0140-6736(96)91080-6

10 Schneider G. Oxygen supply in rural Africa: a personal experience. Int J Tuberc Lung Dis. 2001;5:524-6. Medline: 11409578

11 Turnbull H, Conroy A, Opoka R, Namasopo S, Kain K, Hawkes M. Solar-powered oxygen delivery: proof of concept. Int J Tuberc Lung Dis. 2016;20:696-703. Medline:27084827 doi:10.5588/ijtld.15.0796

12 Duke T, Frank D, Mgone J. Hypoxaemia in children with severe pneumonia in Papua New Guinea. Int J Tuberc Lung Dis. 2001;5:511-9. Medline:11409576

13 Laman M, Ripa P, Vince J, Tefuarani N. Can clinical signs predict hypoxaemia in Papua New Guinean children with moderate and severe pneumonia? Ann Trop Paediatr. 2005;25:23-7. Medline:15814045 doi:10.1179/146532805X23317

14 Sa'avu M, Duke T, Matai S. Improving paediatric and neonatal care in rural district hospitals in the highlands of Papua New Guinea: a quality improvement approach. Paediatr Int Child Health. 2014;34:75-83. Medline:24621233 doi:10.1 179/2046905513Y.0000000081

15 Graham HG, Ayede AI, Bakare A. Oxygen for children and newborns in non-tertiary hospitals in South-west Nigeria: a needs assessment. Afr J Med Med Sci. 2016;45:31-49.

16 Duke T, Graham H, Peel D, Izadnegahdar R, Duke C. Solar powered health care. Int J Tuberc Lung Dis. 2016;20:572-3. Medline:27084807 doi:10.5588/ijtld.16.0210

17 Peel D, Neighbour R, Eltringham RJ. Evaluation of oxygen concentrators for use in countries with limited resources. Anaesthesia. 2013;68:706-12. Medline:23654218 doi:10.1111/anae.12260

18 Peel D, Howie SRC. Oxygen concentrators for use in tropical countries: a survey. J Clin Eng. 2009;34:205-9. doi:10.1097/ JCE.0b013e3181bb1329

19 Duke T, Peel D, Graham S, Howie S, Enarson PM, Jacobson R. Oxygen concentrators: a practical guide for clinicians and technicians in developing countries. Ann Trop Paediatr. 2010;30:87-101. Medline:20522295 doi:10.1179/14653281 OX12637745452356

20 Hill SE, Sanneh M, Jallow M, Peel D, Njie M, Weber M, et al. Oxygen for treatment of severe pneumonia in the Gambia, West Africa: a situational analysis. Int J Tuberc Lung Dis. 2009;13:587-93. Medline:19383191

21 World Health Organization. Technical specifications for oxygen concentrators. http://apps.who.int/medicinedocs/en/d/ Js22194en/. Geneva: WHO; 2015.

22 World Health Organization. Oxygen Therapy for Children. 2016. Geneva: WHO. Available: http://www.who.int/maternal_child_adolescent/documents/child-oxygen-therapy/en/. Accessed: 1 March 2016.

23 World Health Organization. Hospital Care for Children: guidelines for the management of common illnesses with limited resources. 2nd ed. 2013. Geneva: WHO. Available: http://www.who.int/maternal_child_adolescent/documents/ child_hospital_care/en/. Accessed: 2 March 2017.

24 Duke T, Graham SM, Cherian MN, Ginsburg AS, English M, Howie S, et al. Oxygen is an essential medicine: a call for international action. Int J Tuberc Lung Dis. 2010;14:1362-8. Medline:20937173

25 Morrissey B, Conroy N, Estelle A. Effect of solar panels on in-patient paediatric mortality in a district hospital in Sierra Leone [abstract]. Arch Dis Child. 2015;100 Suppl 3:A114.1. doi:10.1136/archdischild-2015-308599.253

26 Were WM, Daelmans B, Bhutta AZ, Duke T, Bahl R, Boschi-Pinto C, et al. Children's health priorities and interventions. BMJ. 2015;351:h4300. Medline:26371225 doi:10.1136/bmj.h4300 